\title{
A framework for systems and network management ensembles
}

\author{
E. D. Zeisler \\ The MITRE Corporation \\ 7525 Colshire Drive; MS W549; McLean, VA 22102; USA \\ Phone: (703) 883-5768; FAX: (703) 883-5241; \\ ezeisler@mitre.org \\ H. C. Folts \\ Defense Information Systems Agency \\ 10701 Parkridge Boulevard; Reston, Virginia 22091-4398; USA \\ Phone: (703) 487-3332; FAX: (703) 487-3351; \\ foltsh@cc.ims.disa.mil
}

\begin{abstract}
A richness of systems and network management technology has been defined by standards. The ensembles method developed by the Network Management Forum (NMF) joins the standards with operational functions used by the enterprise resource manager. In order to ensure that the total enterprise is considered, a framework is required that will tie NMF ensembles to a wider (scalable) management mission. This paper sets out a framework for selection of management ensembles.
\end{abstract}

\section{Keywords}

Domain, ensemble, managed objects, scenario, Telecommunications Management Network (TMN) 


\section{BACKGROUND AND OVERVIEW}

\subsection{Problem statement}

An Ensemble is an OMNIPoint NMF reusable, implementation specification. This specification is made up of requirements, scenarios, and managed objects, plus references to standard information models, and conformance test descriptions.

Ensembles are written and approved through the NMF Ensembles Working Group (EWG). There are a number of implemented OMNIPoint ensembles, which rely on the Telecommunications Management Network (TMN) ITU-T standards. For example see document Forum 017, 1992, Reconfigurable Circuit Service - Configuration Management.

In fact, to provide a detailed representation for both communications and managed data, an ensemble includes actual CMIS/CMIP commands, which access one or more managed objects. The method has successfully provided specifications to the subcontractor(s) who build from them. However, current ensembles provide a solution to a specific (limited), network management problem. The method cannot enable one ensemble to be related to another in a cohesive manner; nor does it provide an enterprise context for the selection or build of multiple ensembles. Enterprise in the functional sense, encompasses: (a) realtime ITU-T X.700 series of specific management functions (for performance, security, accounting, configuration or fault management); as well as (b) non realtime planning, engineering and service provisioning, among others.

\subsection{Concept and background}

As shown in Figure 1, an ensemble, by itself, can provide a window into the real operational functions like alarm surveillance, and into the managed resources, for a class of equipment. The ensemble matrix shows types of managed resources on one axis, while the management functions are shown on the other axis. One or more of these resources and functions can be used in an ensemble to support a specific business objective.

\section{Concept}

The proposed new method (dotted lines in Figure 1) will represent ensemble 'sets' as domains or partitions for the delegation of management responsibility according to enterprise policy. Earlier work has demonstrated how very large-scale distributed systems can be managed using domain and policy concepts [ESPRIT, 1993]. To this end, controlling interfaces is predominantly a matter of structuring different types of organizations, geographic areas, groups of users, or managed technology into domains. Figure 1 shows how ensembles can be further characterized: by policy, domains, services, and features. In general, a service or feature could be provided in some domains, but not others. The point is that, with hundreds of networks, equipment types, services, features and management functions, an ensemble specification/build must be tailored to a domain of interest.

\section{Background}

The genesis of the framework comes from implemented OMNIPoint 0 RCS ensembles. These ensembles led to the British Telcom CONCERT system, which operates with an endto-end view of a complete network, and further, enables interworking with a range of network management systems, as exemplified in Newbridge Network's ConnectExec system for managing a T1 MUX [Gamble, 1993; Newbridge, 1994]. To develop our domain-based framework, we examined the shared management knowledge (SMK) utilized by the just-mentioned implementation systems; we selected and added objects (or object subclasses) for what we call the 'core managed objects'. Experience has shown that it is better to define a core Man- 
agement Information Base (MIB) first, containing only essential objects; later, if experience demands, other objects can be added [Rose, 1991].

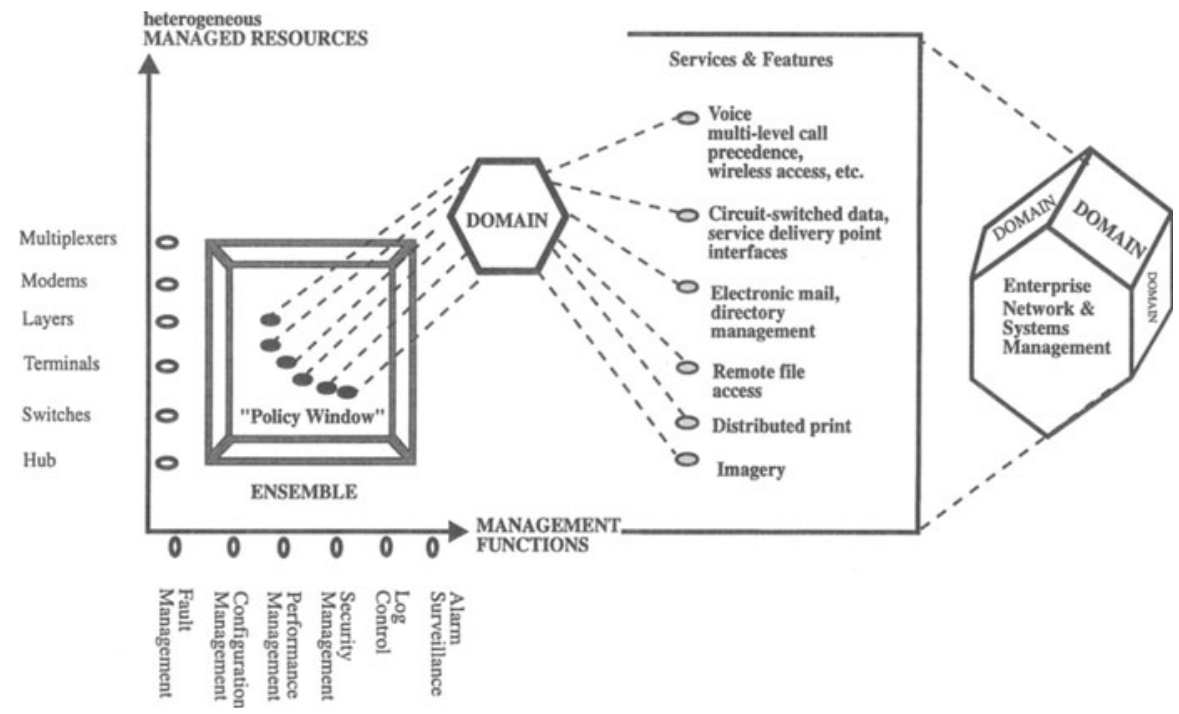

Figure 1 Domain Concept for Ensemble Selection.

Table 1 shows how the designer can associate policy, core managed objects, and a functional area, in this case performance management, for a given domain [Newbridge, 1994; Forum 022, 1992]. The chart shows:

- A 'service description' legend at the upper left, providing a domain association,

- Columns - 'core' Managed Objects (MOs) that can be used in managing the voice service;

- Rows - the performance policy; each cell relates a policy to the objects; the objects will contain the behavior and attributes required for policy institution; and,

- The U (update) and R (retrieval) notations, which set the stage for more detailed specification (i.e., specifying the low-level protocol operations to access objects).

To summarize, the 'core' objects, packaged along with the standards that guide or restrict the use of those objects, not only can express the union of objects across domains, but also can be coupled with a strategy for intra-domain coordination.

\section{Criteria}

The standard core objects are expressed in OMNIPoint 1 syntax. Given a base set for all domains, any ensemble must provide one or more of the 'core' objects. Other criteria follow:

- Monitoring and control - some core objects will be used by other objects, i.e., notifications pass through the sieve (discriminator object) to create events (event records object) that can be logged (log object) or reported. It's possible that only one object (the system object) would be used, as the top of a hierarchy that defines additional subclasses, for layer management. 
Table 1 Sample of Core Managed Objects Related to Corporate Policy

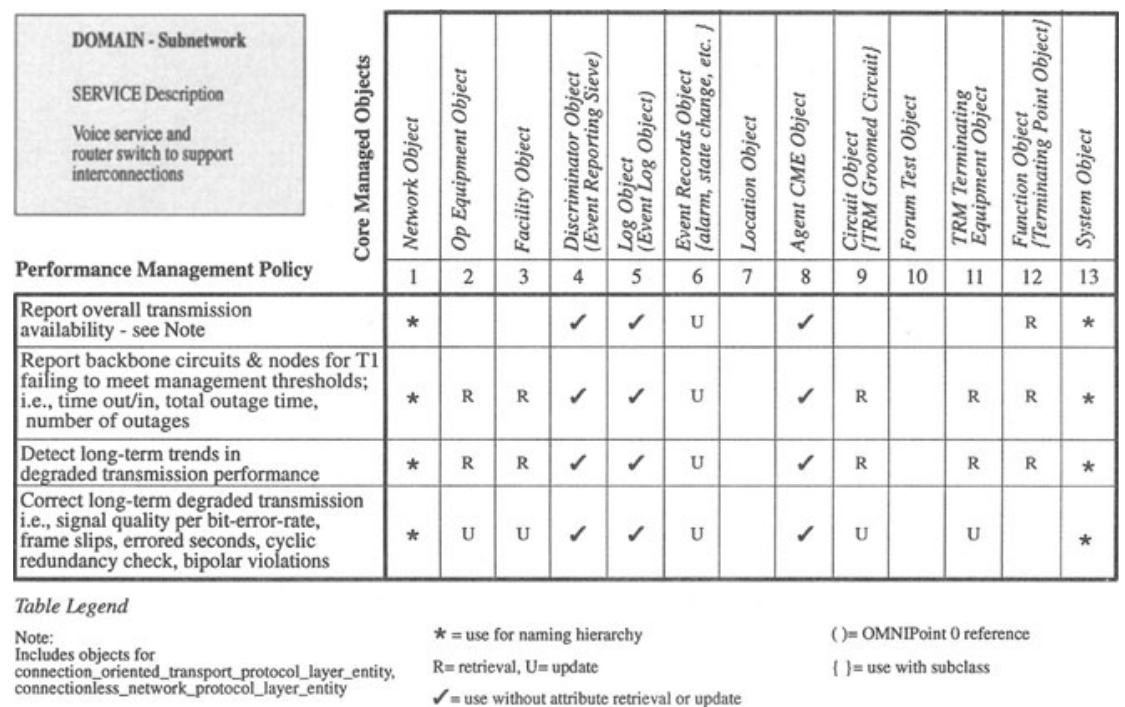

- Extensions - other MOs could be added to an ensemble like the function object, service object, policy object and security object. For instance, the service object in the draft 'Customer Administration Configuration Management' ensemble is used for voice service types including bearer, supplementary and teleservice. A rule of thumb is that the lower-level managers in a domain hierarchy may have a limited number of core object extensions (data attributes/values).

Of relevance, our framework does NOT attempt to provide a step-by-step procedure for developing an ensemble.

\section{Goal}

As mentioned, the NMF ensembles being developed describe managed objects using a specific small (limited) management task area. The domain framework intends to enable development of open systems in a wider management task area by defining a 'domain (partition)' as a small task area and correlating multiple 'domains'. Here, relationships of domains are hierarchical, and some domain management facilities are needed for negotiations between domains.

\subsection{Terms and concepts}

Overall, a taxonomy of domains is vital to our framework. Domains are sets of objects/entities/things that may be logical or physical. For the purposes of this paper, we are primarily interested in management domains, which restricts this generic definition to the extent that the objects/items contained within the set are all subject to the same management agent(s). Another way of putting this is that the domain defines the span of control or sphere of influence of management. Additional requirements (e.g., the managed objects must be finite, named/ catalogued, etc.) have been set forth elsewhere. 


\section{DOMAIN HIERARCHY}

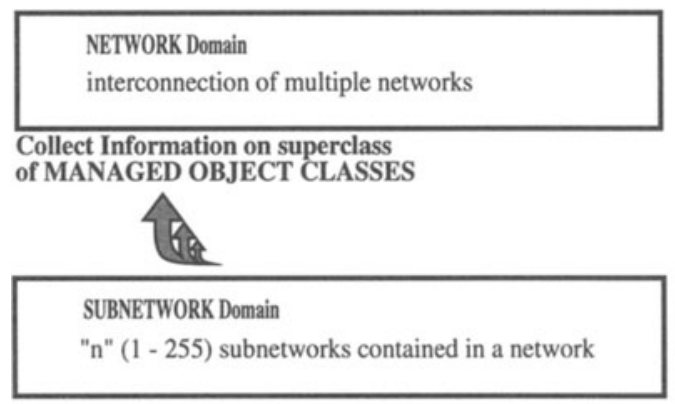

Pass information on 'CORE' MANAGED OBJECTS

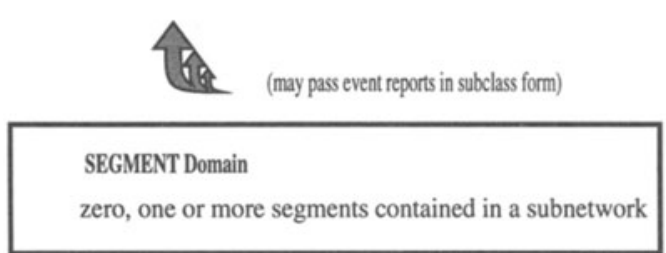

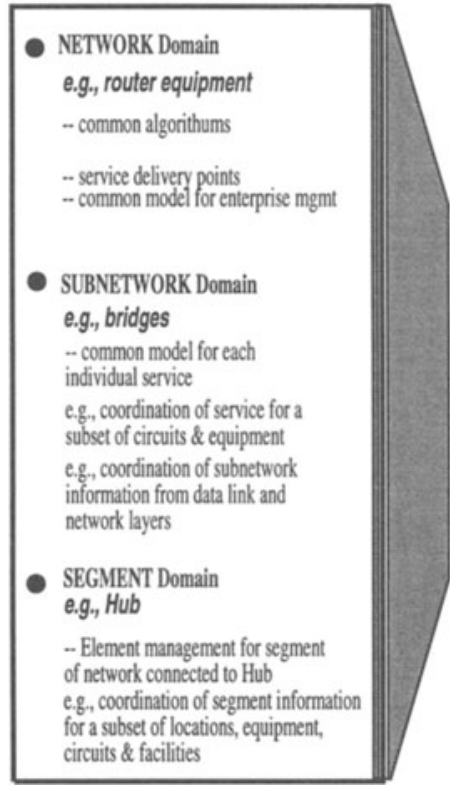

Figure 2 Domains for Connection-oriented Networks.

\section{Physical domains}

There are acknowledged differences on how to standardize and implement domains in the marketplace (ISO CD 10164-19.2) [Moffet, 993]. As used herein, a physical domain consists of a set of real-world objects within a boundary. An example of such a physical domain is all of the computers and peripheral devices contained within one building to comprise a computer system. Another physical partitioning concept could use a domain 'triple' set (networks, subnetworks, segments) for managing different types of switches: one domain manages a public branch exchange (PBX); another domain manages a router; the domains are interconnected to support an end-to-end network management service. Section 3 will apply this triple in a scenario. Figure 2 illustrates the triple domain hierarchy. The relationship of domains in a hierarchy reflects a tailored, open path within a wider networked environment.

\section{Logical domains}

Logical domains may be broken down in as many ways as one may logically group either real-world objects (or representations of real-world objects) or logical resources. To this purpose, a domain hierarchy could be logically partitioned as follows:

- Disjoint or independent - a set of domains that do not interact in any way with each other;

- Overlapping - at least two management domains exist-each containing its set of managed objects, but some or all of the objects in each set (where the two domains intersect) are subject to the management of each of the domains; and,

- Nested - an outer domain exists which contains its set of managed objects and some of its objects may be within an inner domain; the objects in the inner domain are subject to the 
management of that domain, but they are also still subject to the control and are owned by the outer domain.

Further, there must be rules for setting up domains: for instance, a rule might state there can be only one domain manager (coordinator) for each partition in a nested domain.

\section{FRAMEWORK MODELS}

Besides domain design, different models may provide new perspectives for ensembles selection:

- Service model - in this model, requirements for managing components are broken out by services, such as mail, software distribution, printing, remote file access, etc.;

-Work flow model - this model shows organizational requirements based on management controls, input/outputs, processes, subprocesses, or systems; and,

- OMNIPoint business model - this is an administrative view of the managed components for the customer, supplier and service provider; associated with ITU-T TMN interface points [Q821, 1993]

The two models that are described herein exploit multiple perspectives for the framework: the first is a generic network model, which describes Connectivity at a high level; the second, is a model for the ITU-T TMN interface points (see Section 3.3).

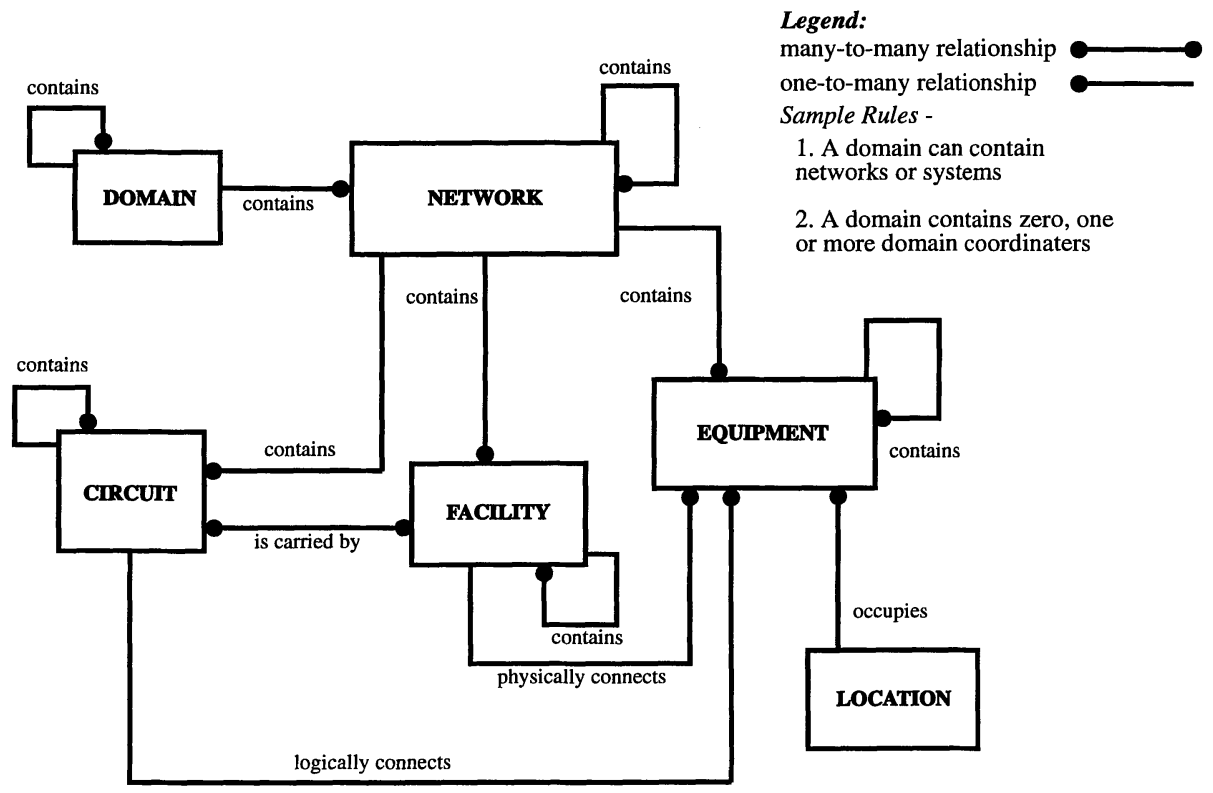

Figure 3 Generic Model of the Network. 


\subsection{Generic network model}

Figure 3 uses a subset of the 'core' objects for describing connectivity. This kind of generic model of connectivity is essential to the generation of uniform operations, planning, engineering, service provisioning and maintenance. The specific resource properties and attributes for each Figure 3 entity are specified in the OMNIPoint NMF Libraries.

First, the model can be used to describe connectivity for any number of domain types. That is, the entity-relationship diagram relates each domain to its component networks/subnetworks/ segments, circuits, facilities, equipment, and locations.

Second, besides populating the model for network connectivity, it could be expanded for systems information: the domain entity could contain a system entity, which is separate from the network entity, and relates to other entities that are used for system management.

Third, the model identifies the building blocks for a component (resource) hierarchy for an ensemble. Ultimately, service-users may control and observe the values of the attributes of these resources. An example is given next to show the distinction between circuit and facility, as used in the model [Kennedy, 1991].

The circuit managed object refers to a connection that is independent of the means of carrying the signal. Instances of this managed object carry information from point to point and preserve its content. The bandwidth (information/data rate) of a circuit may be known, fixed or variable.

The facility managed object refers to the physical means of carrying a signal; e.g., a connection composed of different media (coaxial or fiber cable) in an ordered sequence with intervening equipment (for optical-electrical conversion, reshaping, or regeneration). The signal rate and medium of a facility may be known and fixed and is implied by the facility type. Thus, the bandwidth of a facility is not dynamically alterable. Facilities are used to carry circuits:

- one facility object instance can carry many circuits;

- one circuit object instance may be carried by many facilities;

- a facility physically connects to one or more equipment's; and,

- a circuit logically connects to one or more equipment's.

\subsection{Modeling tools}

The Integrated Computer Aided Manufacturing (ICAM) Definition (IDEF) graphic representations of the network model employed an IDEF-based modeling tool, since the IDEF family of standards is nonproprietary and public domain:

- the generic network model plus an expanded model (template) with the business rules for domain activities and entities, were created using IDEF1X data modeling syntax;

- high-level management functions were decomposed using an IDEF0 function modeling tool [DISA, 1993].

\subsection{Outcomes}

Requirements were modeled to develop a generic template for all domains. The full template includes a characterization of domain activities and an operational model (i.e., domain manager, shield, domain agent, and target resources). A shield is an intelligent control between the manager and agent, which hides nonrelevant details from the manager. In general, the shield supports intelligence for visibility control, computation, aggregation and/or transformation [ESPRIT, 1993]. 
Modeling supported a kind of 'intermediate analysis', or translation, for the sampled requirements. Further, the IDEF rigor imposed should ensure consistency for our next phase, which will produce a prototype for domain ensemble(s).

\section{DOMAIN SCENARIOS}

Now that we have described our framework and goals for the OMNIPoint Network Management Forum EWG, we will look at how we actually used the concept. A typical domain requirement for a large packet-switched data system would be 'the service shall be capable of supporting a hierarchy of logical subnetworks with independent addressing and management domains. At least three levels of hierarchical networks, with at least 256 logical subnetworks at each level, shall be supported'.

\subsection{Voice service for organization independent views}

In this particular scenario, the Figure 2 domain breakout (networks, subnetworks, segments) is applied to meta-management of voice communications management systems. The domain triple, is compatible with other models, in particular for TMN administrative roles (service provider, subscriber). Figure 4 has three rings: the outer ring represents the End-User subnetwork domain, the middle ring shows the Local Exchange Carrier (LEC) subnetwork domain, and the inner ring represents the Interexchange Carrier (IC) backbone subnetwork domain. The views (labeled one, two, and three) illustrate activity for the different players, respectively:

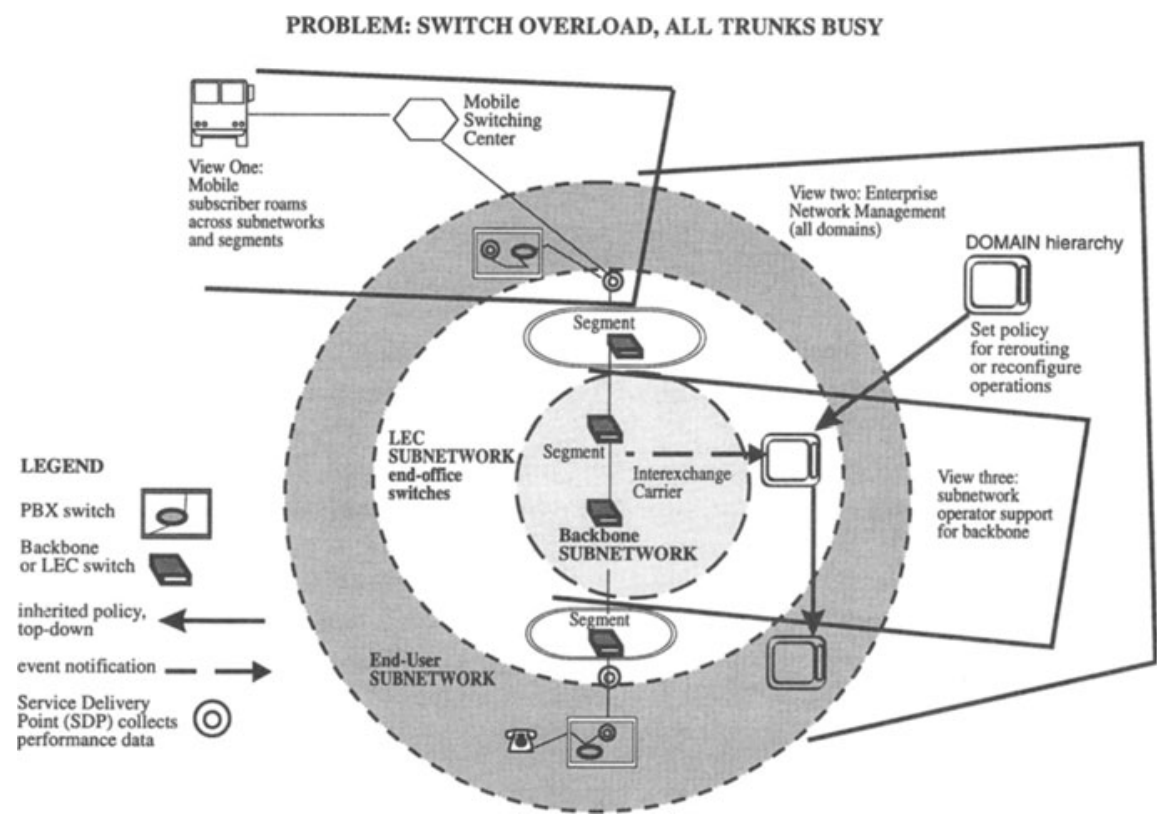

Figure 4 Voice Service Scenario for Performance Management. 
- view one - a customer's (subscriber) view from the end-user subnetwork;

- view two - a service provider's enterprise view of the switching network and all domains;

- view three - an internal operator's view, in this case providing support in a service provider role, for the backbone subnetwork domain.

The Figure 4 partitions (rings) and organizationally-independent views were used, to apply the triple (network, subnetwork, segment) domains design. Of benefit, overlapping organizationally-independent domains enable scalable management:

- ability to institute rights over another manager's domain (constraints);

- flexibility for inherited behavior for a sphere of interest; and,

- ability to harmonize management policy for heterogeneous, distributed resources.

That is, a user could be supported by:

- whichever domain operator controls the switches/trunks at the time a problem occurs

- whichever domain triggered a network fault, performance or accounting error.

In general, any of the Figure 4 switches could be involved in management activity, since they constitute a component hierarchy for the end-to-end switched voice service: (a) either of the end-user PBX switching nodes; (b) either of the LEC subnetwork switching nodes; and (c) either of the backbone switches for the IC switching node.

\subsection{Sample scenario: voice service and performance management}

Again, Figure 4 illustrates just one possible domain scenario. The performance problem (all trunks are busy) is due to a switch overload in the backbone subnetwork domain. Consequently, problem management impacts the mobile subscriber and must be resolved by the backbone subnetwork operator. Note that because all circuits are busy, the subscriber can't call in the problem, and the trouble $\underline{\text { must }}$ be detected by the network management system.

\section{Domain interaction}

First, in the innermost ring, a state notification or fault is generated concerning service to the mobile subscriber. Simultaneously, a user error message to a domain management station in the outer ring (end-user subnetwork) associates the call problem with one of the backbone switches, and further identifies a cause code.

$\underline{\text { Second, }}$ to resolve the problem, the view analysis will coordinate among:

- the End-User, LEC, and backbone subnetwork switch domains for performance and state information; and,

- the enterprise network manager and backbone subnetwork domain, for backup plans for bandwidth/signal/channel allocations.

Third, something must happen in the backbone ring to resolve the problem.

- the backbone domain manager could perform rerouting operations in the backbone subnetwork domain (i.e., using view three); and,

- a dynamic rerouting solution could require coordination between views two and three.

Last, through the hierarchy of domain managers, the subnetwork and segment domains affected will receive knowledge of a reconfiguration. Further, the coordinated information could be used not only to resolve the performance problem, but also to adjust customer usage billing. 


\section{Ensemble requirements}

Intelligence in the domain agent is needed, to correlate performance and state information. Also, there can be policy related to thresholds for blocked subscriber calls or related to who needs to be notified (e.g., the stations at the access switch).

Ordinarily, in realtime when a trunk group becomes unavailable due to congestion and there is limited available resources, a mobile user's calls will be dropped based on user priorities (multilevel precedence and preemption activity). Thus, a series of dropped calls or error messages (call incomplete) could occur until the threshold for critical congestion is exceeded.

\section{Domain coordination functions}

As shown in Figure 4 (with workstation screens), each subnetwork domain 'manager' has capability to institute end-to-end service policy and policy for reporting performance, as directed by the enterprise network domain manager. For instance, the domains will regularly:

- pass performance logs between domains with overlapping managed resources;

- use alternative routes only as short-term solutions, according to policy; or,

- send performance event reports for long-term trend analysis; these could be collected at the different Service Delivery Points (SDPs) in the network, i.e., at the interface between the customer and service provider.

\section{Service Delivery Point requirements}

- Filter collected statistics on degraded transmission performance, and,

- Collect network statistical data on traffic flow.

\section{Benefits}

In summary, the domain concept can enable the operator directly responsible for resolving a problem to become involved, rather than going through intervening 'organizational domains'. Note that getting a window into service provider subnetworks or internal organizations will require setting up service level agreements [Moffet, 1993].

\subsection{Model for Telecommunications Management Network interface points}

Figure 5 maps our scenario to the TMN architecture (M.3000 series of recommendations from ISO/ITU-T). The maturation process of the TMN standards will continue to accumulate importance for service providers and network equipment vendors for the remainder of this decade. Therefore, a benefit of the domain framework for Systems and Network Management Ensembles is that it could be used to set goals for integrating TMN into the enterprise requirement.

Essentially, a TMN is a network to provide surveillance and control of another network. The management network may be separate from, or share elements with the network it controls. Figure 5 references the scenario domains, to identify standardized TMN interfaces.

Function blocks for interfaces are [Shrewsberry, 1994]:

WSF - workstation function to interpret TMN information to the human user

OSF - operations system function to process information related to management

QAF - Q adapter function for protocol conversion to a standard TMN interface

NEF - telecommunications functions for the network element (managed device), including the MIB and associated management applications

MF - mediation function to store, adapt, and filter detailed information between an OSF and a NEF or QAF. 
A Reference Point (RP) classified for the message communication between any two function blocks is [Shrewsberry, 1994]:

f - attachment for a workstation function; used here as an X Windows interface to a human user

m - class between a QAF and its non-TMN managed resources; typically an older telecomtype interface like Bellcore's Translation Language 1 (TL-1) or the Telemetry ByteOriented Serial (TBOS) protocol

q - a class between an OSF, QAF, MF, or NEF for standardized interoperability (e.g., between the network management, element management, and managed element) or between pairs of each; a q3 is a fully standards-compliant interface that uses a CMIP manager/agent pair on OSI protocol stacks. A qx is a 'not quite q3', where conformance problems arise in the network element layer (between switches in the subnetworks and the domain manager) or where the embedded system is small or uses SNMP

$\mathbf{x}$ - a class for providing interoperability between an OSF and a similar function in another management network; an interface between the administrative domains (service providers and customers) uses core objects to pass event reports or invoke maintenance.

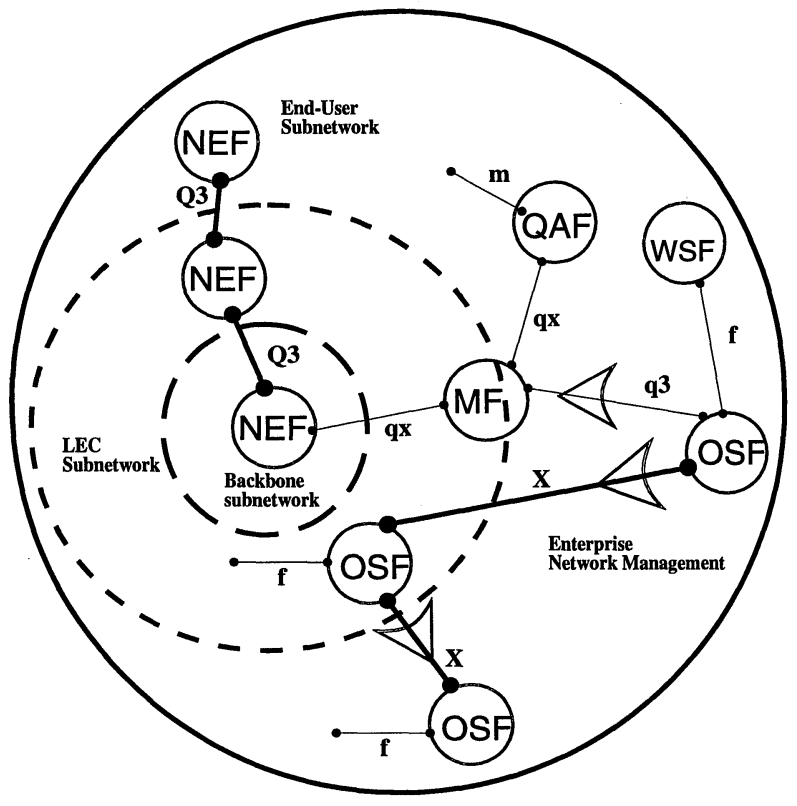

Figure 5 A Global Set of Requirements.
LEGEND and ITU-T
M.3100 TMN options

Q3 - data comm interface

$X$ - data comm interface to carriers, customer f - X Windows interface

m - reference point (RP);

protocol gateway for voice protocols qx - RP, event reports

q3 - RP, shield mediation to domain manager

Shield $<$

In Figure 5, upper case and emboldened lines, distinguish an interface from a RP. A RP becomes an interface when it occurs at a location requiring data communications between hardware elements. Significantly, all the managing software in a layer of the TMN architecture, resides at the application layer of the OSI reference model; the agents and services are not tied specifically to the underlying layers of the OSI protocol stack. 
The Figure 5 shields separate managing and managed components (e.g., hardware devices) across domain boundaries; as a result, the manager works through the shield, to invoke/forward SMK to another domain or target element management system. For instance, 'shield' computations (located in the agent) could centralize bit-error-rate (BER) calculations.

\section{CONCLUSIONS}

As network connectivity grows, there is a need to extend the scope of management from a few nodes to a global environment, where many networks are interconnected. We have designed a framework to make open systems development in a wider task area easier and cheaper. Service providers invest immense amounts of money with suppliers for technology that will support their service strategies; therefore, we predict significant cost savings if a domain hierarchy is adaptable to managing services for heterogenous, multi-vendor devices.

odeling for ensemble negotiations between domains focuses on how to effectively share management knowledge across provider and customer networks. Further, the framework enabled us to identify three potential candidates for future prototyping and ensemble specification: (a) Customer interfacing TMN domains for intelligent correlation of switch faults; (b) Performance Management Ensemble for correlating BER across domains; and, (c) Customer interfacing TMN domains for intelligent correlation of trouble tickets.

In the next phase, we plan to prototype parts of the triple domain set in a physical design for the optimal number and type of domains. The prototype will use the generic network model to specify logical connectivity; a shield and domain MOCs will be identified, for policy institution. In prototyping TT and/or fault correlation, we intend to investigate the impact of the management protocol, as regards how many managed nodes the domain coordinator (station manager) can manage. It is assumed that coexistence between SNMP and CMIP is required, and both COTS and ensemble (new) development code will be used.

\section{REFERENCES}

Defense Information Systems Agency (DISA) (1993) Communications System Network Management: Network Flow Diagrams. AT\&T/Seta Team, Reston, VA.

ESPRIT Project No. 5165 (1993) Distributed Open Management Architecture in Networked Systems (Domains). Deliverables D2a, V1.0 and D3, V1.0.

Gamble, R. (1993) Generic Agent CME. British Telecom CONCERT OMNIPoint 0. Belfast Engineering Center, Ireland.

Kennedy, T.W., Riegner, S.E.M. (1991) An Object Oriented Model for the Operations, Administration and Maintenance of FAA Telecommunications. MITRE Corp., McLean,VA.

Moffet, J.D., Sloman, M., Twidle, K.P., Varley, B.J. (1993) Domain Management and Accounting in an International Cellular Network. IFIP Transactions III.

Newbridge Networks (1994) MainStreet Connect Exec, Technical Reference. Rel 5, Generic SBL115.

NMF OMNIPoint Forum 017 (1992) Reconfigurable Circuit Service: Configuration Management Ensemble V1.0., Morristown, NJ.

NMF OMNIPoint Forum 022 (1992) NM Forum Mapping from Release 1 to OMNIPoint 1. Morristown, NJ.

Q.821 CCITT Rec and NMF OMNIPoint (1993) Strategic Framework. Morristown, NJ.

Rose, M.T. (1991) Network Management is Simple: you just need the "right" framework! IFIP Transactions II.

Shrewsberry, J.K. (1994) TMN in a Nutshell, V1.01. WilTel, Tulsa, OK. 


\section{BIOGRAPHY}

Elizabeth D. Zeisler

Liz Zeisler, a lead scientist with the MITRE Corporation, has over 20 years of lifecycle development experience in information systems, database and network management. She has degrees from Cornell University (B.F.A.), Computer Processing Institute (A.A.), State University of New York at Buffalo (M.E.) and American University (M.S.).

Harold C. Folts

With 35 years experience in telecommunications, Hal Folts currently serves as a senior systems engineer for network and systems management applications in the Defense Information Systems Agency. He has been involved over the past years with the development of many international standards for data communications and open systems. He has a BSEE from TriState University and an M.S. in Systems Management from the University of Southern California. 